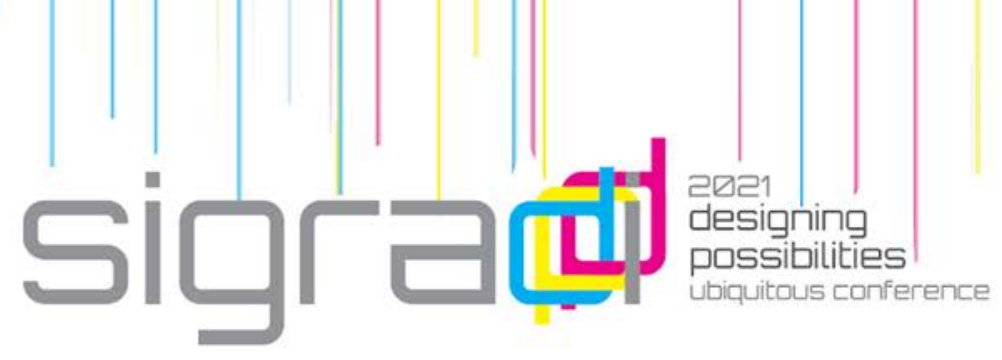

\title{
Hybrid Carbon Fiber and Jute Fiber Textile Bone Stool: Integrative Fabrication Method of Weaving and 3D Printing
}

\author{
Berfin Evrim \\ University of Calgary, Canada \\ berfin.evrim@ucalgary.ca
}

\begin{abstract}
The structural properties of Fiber Reinforced Polymers (FRP) encourage designers and architects to use textiles as a load-bearing architectural material to create lightweight and strong structures. Manufacturing techniques of FRPs are mostly concentrated on the molding method. This method requires an extra mold fabrication that causes waste of material. This study focuses on integrative weaving and 3D printing fabrication methods, which emphasize the lightweight property of the material. This integrative method avoids excessive material waste during fabrication by using an additive approach. 3D printing on textiles prevents significant deformation in a specific direction of the fabric instead of using any kind of synthetic resin for stiffening the fabric. Additionally, structural behavior simulation allows designers to understand the different loading conditions and maximize the strengths of each textile design by adding more material where it is needed for possible architectural applications.
\end{abstract}

Keywords: Stool Design, Bone Analysis, Textile Load Simulation, Weaving, 3D Printing

\section{Introduction}

Fibers present significant opportunities for the future practice of architecture because of their lightweight and high-strength properties. The ultra-lightweight characteristic of fibers lowers transportation and construction costs. Moreover, carbon-reinforced material can be shaped to a wide range of geometries and interlaced with natural fibers to avoid negative environmental impacts and achieve material and cost-efficiency. This study focuses on integrative weaving and 3D printing fabrication methods to avoid material waste as a result of the conventional molding method.

To add stiffness to fabric, this study suggests 3D printing Thermoplastic Elastomer, which is non-toxic, and plasticizer-free. Thermoplastic Elastomer 
can be a substitute for the synthetic resin to prevent significant deformation in a specific direction of the fabric by stiffening it. The fabrication technique for combining weaving and 3D printing can result in the development of the use of textiles in architecture, which is synthesized with biological principles to create stronger and lightweight structural elements. This study specifically focuses on trabecular bone analysis by SEM imaging for a stool design.

Furthermore, this study investigates different weaving patterns and 3D printing patterns to achieve maximum strength and rigidity of textile design by parametric fabric modeling and hand weaving. To structurally test the textiles, this study presents a digital tool that simulates the structural behavior of 3D printed woven textiles to fabricate a textile that is used for supporting the weight of a person sitting on a stool or chair.

Contributions of this research include several geometric tool paths affecting the stiffness of the textile in combination with the weaving pattern and materials. These toolpaths also include geometry inspired by cow bone structure. Besides, the digital tool is developed in Grasshopper for parametric modeling of textiles (stiffness, geometry, etc.). These textiles can be loaded in this digital tool to simulate their structural behavior. This approach prevents material waste and allows designers to generate fabrics for possible architectural applications in different loading conditions.

\subsection{Background}

To reduce the environmental effect, the hybridization of natural fibers with synthetic fiber plays a significant role. Aakash et al. (2019) explored the hybridization of carbon/jute fabric reinforced epoxy hybrid composites. They suggested that the hybridization of natural fibers with synthetic fibers can allow having high modulus and mechanical strength.

In addition to the hybridization of fibers, analysis of loading conditions is crucial to design strong structures without any waste of material. lida et al. (2014) have designed a zig-zag chair made of carbon-fiber-reinforced polymer. calculate the loading conditions and fold the carbon fiber reinforced polymer fabric for optimized chair design. Neri Oxman (2010) is another designer who analyzes the loading conditions for design optimization. She designed the Beast Project that presents a single continuous surface modulated with Voronoi tessellation according to structural load to function to create a skin and structure. She integrates structural and material performance by analyzing the effect of the loads, curvature, pressure on the thickness, stiffness, density, and pattern of the material.

Furthermore, Martinez (2017) talks about additive manufacturing and mentions the work of Lillian Van Daal, who used a similar approach as Oxman when she was designing the Biomimicry Chair. Van Daal is inspired by the plant cell, and she 3D printed prototypes for flexible and rigid surfaces. She used less 


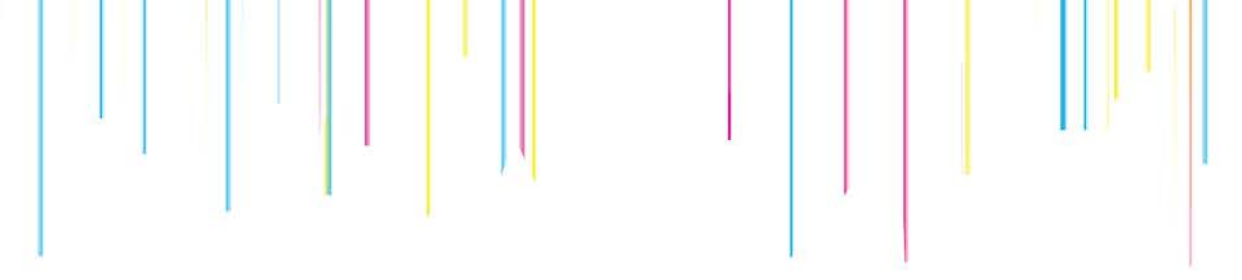

material in horizontal alignment, which is the seating surface, and more material in vertical alignment for structural purposes. She fabricated the chair with 3D printing biodegradable materials.

On the other hand, Estrada et al. (2019) designed a lightweight and hanging chair made of carbon fiber-reinforced polymer and steel bars. They were inspired by Gaudi's models; therefore, they used Karamba 3D for form-finding reasons to apply gravity. They impregnated carbon fiber with epoxy resin. They optimized their design with an integrative approach to design and fabricate by focusing on material research and computational design. In another study, Wang et al. (2020) designed a 3D printed thin bending active formwork for shell structure. They used Kangaroo for form-finding purposes. Then, they printed principle stress lines on the shell and simulated shell deformation under gravity load in Karamba 3D.

Nan et al.(2019) are other researchers that utilized 3D printing but they combined traditional knitting method with digital fabrication methods. They created a formwork that uses 3D printing on fabric as a form generator to cast concrete columns and tiles. Kycia et al.(2020) focus on the self-forming result of 3D printing on textile to create three-dimensional textile surfaces inspired by plant leaves.

\section{Methodology}

After understanding the studies from the literature, I propose a mixed research methodology combining analytic, fabrication, and simulation methods. This research focuses on a stool as a design element for carrying a variety of loading conditions such as horizontal loads (beam-like) and vertical loads (column-like) that must be transferred to the ground.

\subsection{Analytic Methods: Bone Analysis}

This study starts with bone analysis, which is initiated with Scanning Electron Microscope (SEM) images of a cow femur bone to understand the trabecular bone structure and trabeculae alignments in micro-scale and Nanoscale. The bone structure consists of compact bone and spongy bone. The spongy bone is the porous structure that allows the bone to withstand various loads. The specific positioning of the trabecular adds compressive or tensile strength to the bone. 


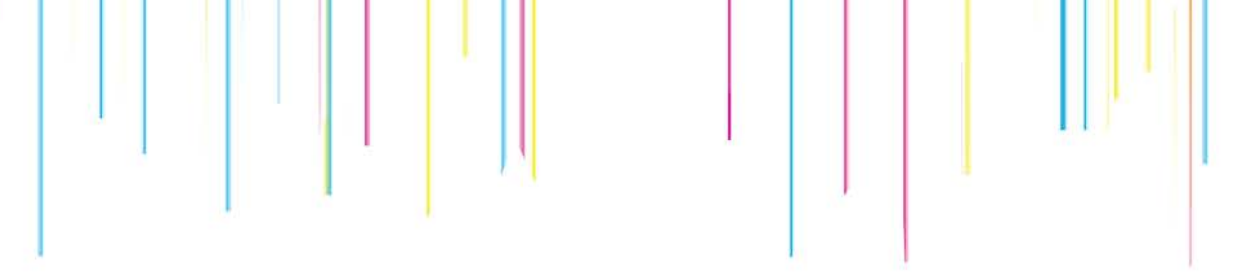

mimicked in a formal shape and structural system. For instance, a roof can be self-standing with a porous and functionally graded material structure.

\subsection{Fabrication Methods: Traditional and Digital Fabrication}

For lightweight structures, the hybridization of natural and synthetic fibers plays a crucial role in achieving the desired strength. Jute fibers have a lightweight, non-toxicity property, high specific modulus, and pollution-free fabrication. The other advantage of the use of jute fiber in the construction industry is easy accessibility with low cost. As well as these benefits, there are some disadvantages of jute fibers that create significant problems such as high moisture absorption, which causes weaker interfacial bond and mechanical properties. A combination of two or more fiber types allows taking advantage of the stronger feature of each material. Synthetic fibers such as carbon fiber have better mechanical properties such as higher tensile strength, stiffness, electrical conductivity, and low thermal expansion; however, they cost higher than natural fiber composites. Therefore, the combination of carbon and jute fibers results in a high-performance structural design at a lower cost.

In this research, 2-ply jute fiber, which is two-strand twisted with $1.3 \mathrm{~mm}$ thickness, is interlaced with continuous $3 \mathrm{~K}$ carbon fiber tow. The hybrid woven fabric is stiffened by 3D printing thermoplastic elastomer (TPE), which is a thermoplastic rubber that contains thermoplastic and elastomeric properties.

\section{Traditional Fabrication: Weaving}

This study focuses on the plain weave and gauze weave that have a weftshifting structure instead of warp shifting. Gauze weave textile is a surface element where a person interacts directly while sitting on the stool. The plain weave textile is used as a structural layer, which combines different ratios of carbon fiber and jute fiber. The main reason for testing different ratios is to understand the amount of carbon fiber that needs to be interlaced with jute to withstand the sitting force of a 250-pound person. All of the woven fabric has a structure of 9 carbon fiber warps and 48 weft elements. The material ratios of carbon fiber to jute fiber are 16/32, 10/38, and 0/48. 


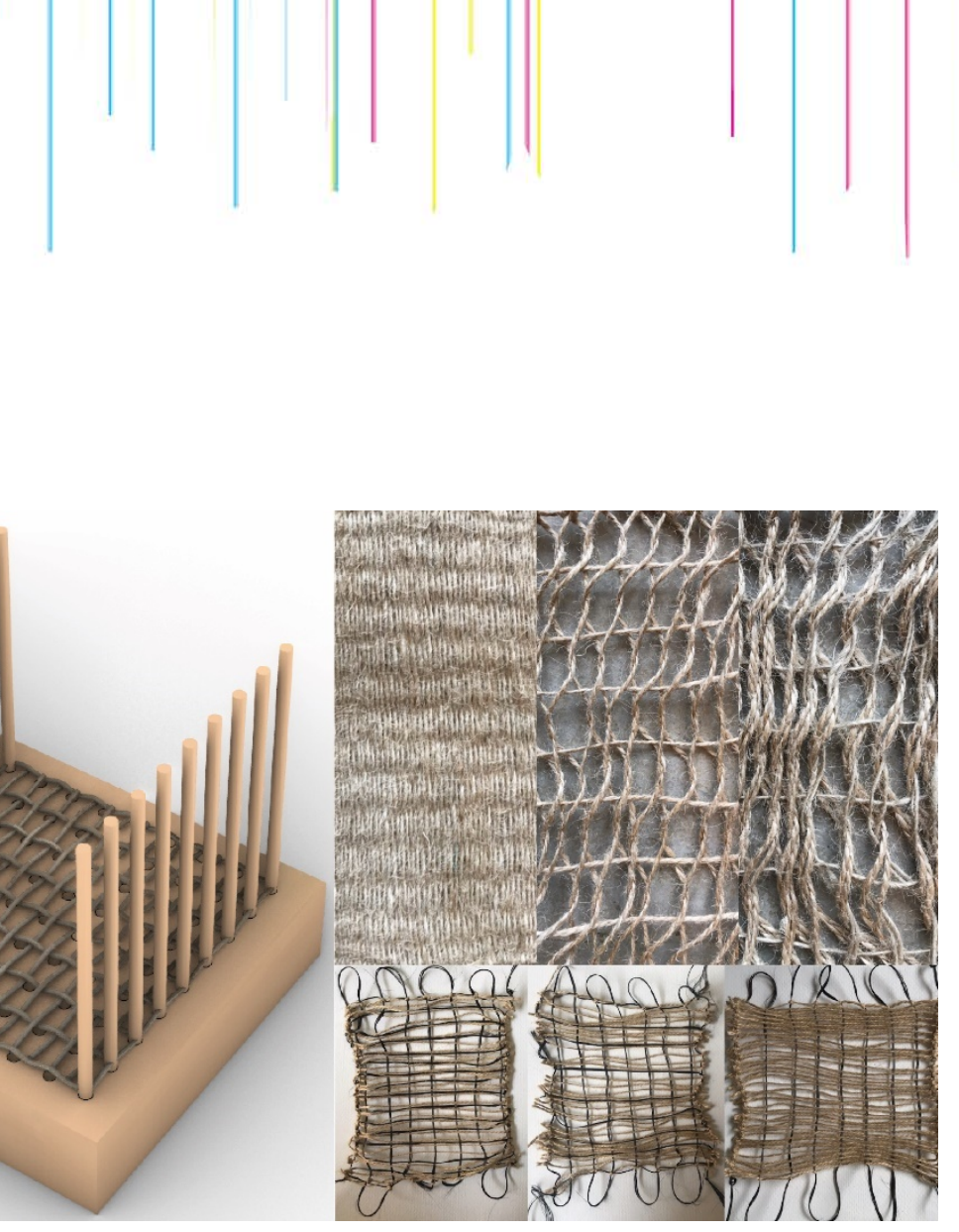

Figure 2. Weaving Loom, Weaving Patterns and Hybrid Carbon Fiber and Jute Textile. Source: Berfin Evrim, 2021.

For the fabrication of textiles, a weaving loom is designed (Figure 2) to fabricate woven textiles. This loom combines the functionality of pegboard and rug loom. The loom contains equally-spaced holes for dowels. Each dowel can be replaced according to the shape and size of the fabric. It can be woven in the z-direction to achieve 3D woven fabric structures. However, this research focuses on a single layer weaving and binding two layers later.

\section{Digital Fabrication: 3D Printing}

The woven fabric is easily deformed in the diagonal direction; 3D printing is a solution to add stiffness and stability to this fabric. The goal of 3D printing on woven fabric is to create a layered fabric system that has sub-layers of printed TPE, which is a sustainable, biodegradable, and cost-efficient material. Since TPE is a mixture of hard plastic and rubber, it decreases the cracking possibilities when the load is applied. The default temperature settings of TPE are $240^{\circ} \mathrm{C}$ for the extruder and $50^{\circ} \mathrm{C}$ for the heat bed. The first layer temperature is increased to $250^{\circ} \mathrm{C}$ and $60^{\circ} \mathrm{C}$. This setting adjustment helps filament to stick well to the fabric.

First, the bed level is adjusted higher to keep the heat-bed closer to the nozzle with the first layer calibration. The fabric needs to be in full tension; therefore, the double-sided tape was used during the 3D printing process. The speed of printing is lowered to $40 \%$, and the infill density is set as $30 \%$. The machine and fabric are preheated before 3D printing. The first layer width is increased to $1 \mathrm{~mm}$ for higher shape accuracy and better adhesion of TPE on the fabric. 
2.3 Simulation Methods: Digital Tool

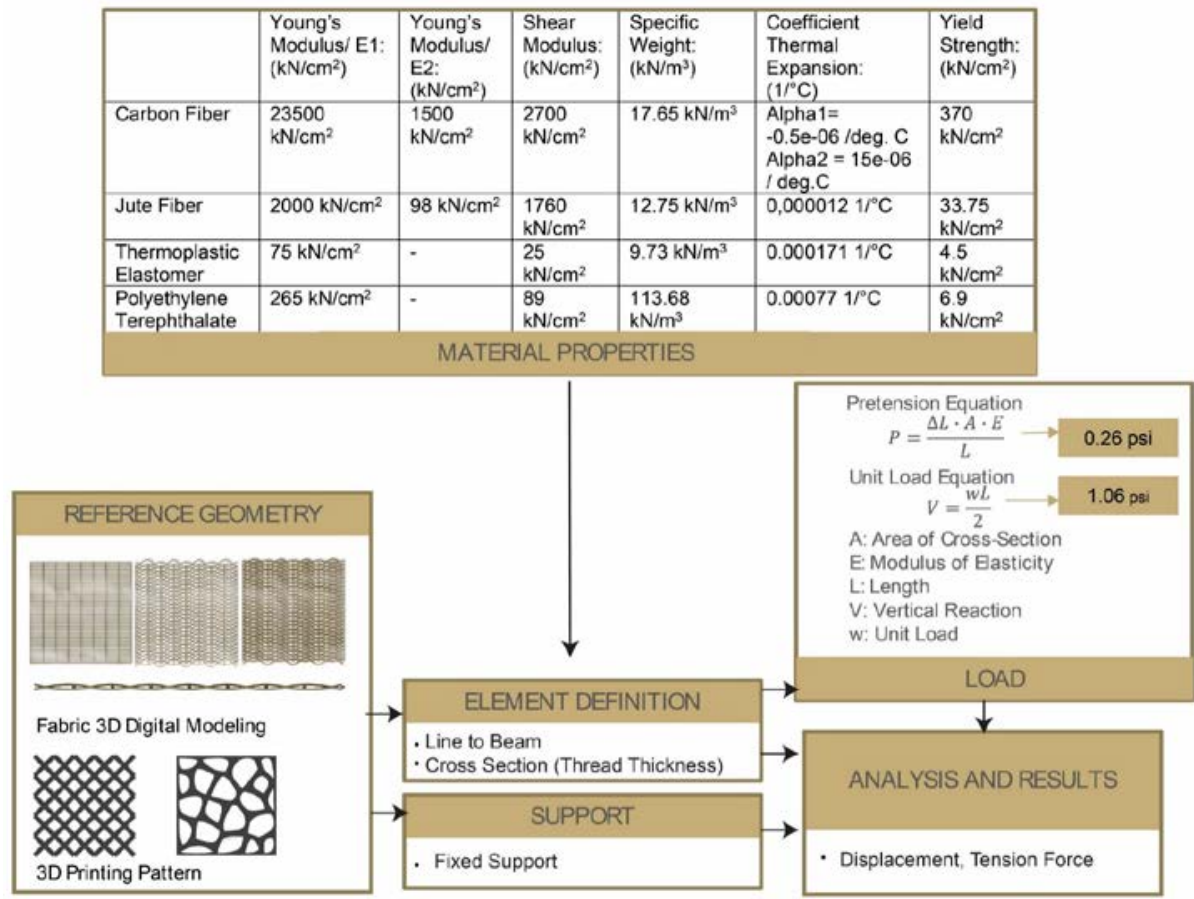

Figure 4. Algorithmic Flow. Source: Berfin Evrim, 2021.

To simulate the structural behavior of textile, a parametric workflow Is created within Karamba 3D, which provides finite element analysis. First, the weaving patterns are modeled such as plain weave, single and double gauze weave structure with parameters to adjust the number of warps and weft, and the fiber thickness. The ratio of carbon fiber and jute fiber can be also determined by the designer in the reference geometry component. The diamond and boneinspired Voronoi 3D printing patterns are also included in the reference geometry, where designers can increase the density and thickness of the pattern with the point attractor component according to the location of load concentration. After modeling the fabrics, each weft and warp and 3D printing patterns are converted into lines and then beam structures.

The beam structure requires inputs of the thickness and the shape of the cross-section and the material properties. The material properties are edited manually for jute, carbon fiber, and TPE because of the limited material library in Karamba 3D. The materials are categorized under two different material types that are orthotropic for jute and carbon fiber and isotropic if any TPE is printed alone.

Later on, the digital tool requires support points, which are selected as fixed supports to avoid translation and rotation in any direction. Then, designers have to determine the load type, amount, and direction. First, the initial strain load is 


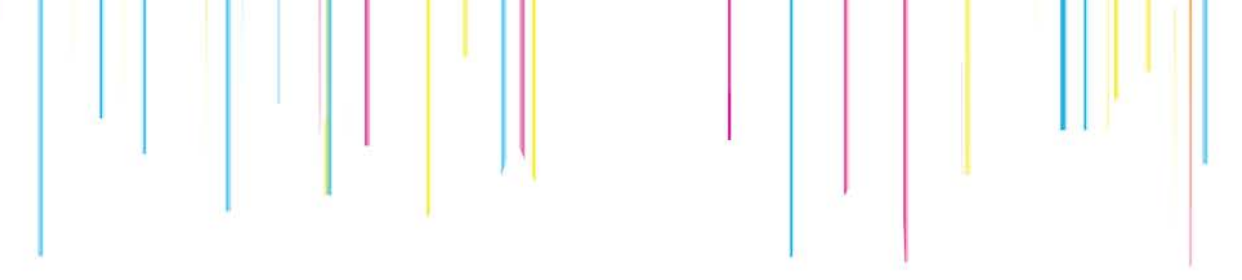

applied to pretense the fabric. The fabric is pre-stressed by the frame, and Karamba 3D simulates the pre-tension effect on the fabric by adding an initial strain load. The vertical unit load is also calculated to simulate pre-tensioned fabrics under vertical load and gravity for more realistic results. The correct calculations of loads are significant to receive a more accurate demonstration from the analysis. Consequently, designers can make better design decisions based on simulation analysis.

In the end, the assembly component gathers the definition of the beam elements, supports, and loads for structural analysis. The simulation calculates the maximum force that each element can bear according to the given safety factor. Additionally, the tool simulates the fabric structures under applied load conditions to show displacement, stress, and utilization. With this tool, it is possible to understand the structural capacity of different traditional weaving patterns, 3D printed plastics in specific toolpaths, and specific carbon fiber tows to jute ratio, making up fabric under given loading conditions. (Figure 4)

\section{Results}

\subsection{Simulation Results}

The goal of the analysis is to avoid any failure with a large amount of displacement and uncomfortable seating surface with too little displacement. Therefore, designed textiles are compared under categories of different weaving patterns, carbon fiber to jute ratio, and 3D printed toolpaths. (Figure 5)

According to simulation results, the plain weave has shown the least displacement, which makes it to be a good weaving pattern for structural purposes. On the other hand, a single gauze weave structure provides a better medium for 3D printing and undergoes the most tensile stress. Thus, the single gauze weave structure is an option for the surface components because it can be easily taped on the heat-bed without having problems with the extruder dragging the fabric. Moreover, the increase in carbon fiber causes a decrease in displacement. The addition of carbon fiber results in higher tensile strength performed by the fabric. In the third category, the diamond shape and Voronoi toolpaths are tested. The toolpaths are modeled on a single gauze woven textile that is made of jute. Even though the results do not show a significant discrepancy, the Voronoi toolpath has less deformation than the diamond tool path, and it can undergo more tensile stress. 


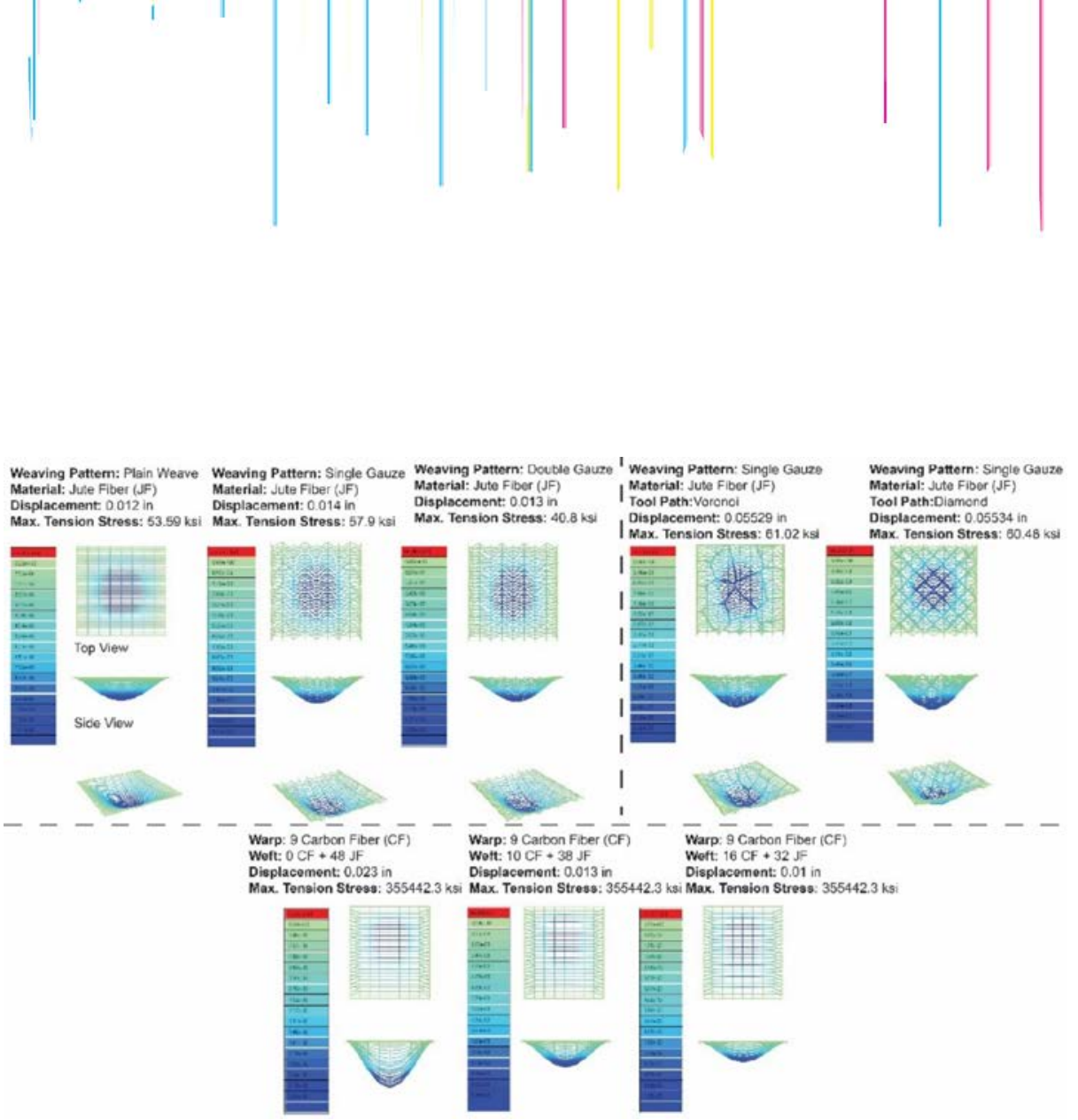

Figure 5. Simulation Results. Source: Berfin Evrim, 2021.

\subsection{Fabrication Results}

The design of the stool consists of two parts that are handwoven and 3D printed fabric, and the stool frame (Figure 6). The woven textile that is stiffened and stabilized with 3D printing techniques has a significant role in providing strength to frame and comfort to the users. Two layers of fabric are overlayed with an orthogonal angle to improve the mechanical properties of the textile. The 3D printing pattern is inspired by bone surface and composed of Voronoi cells. The cells concentrate where pressure concentrates while a person is sitting. The Voronoi pattern is 3D printed on the front and then the backside of each fabric to add stiffness to the textile and prevents distortion on fabric.

The relationship between trabecular alignment and micro-geometries in trabecular bone structure is applied to a stool frame design. The fibers going through the Voronoi cells to attach the bottom of the frame demonstrate the rods in the Nanoscale trabecular structure. The continuous structure of bone is reflected on stool design by using Voronoi tessellation on the frame and fabric, and the strings, which go within the frame, unites the frame structure and fabric top. 

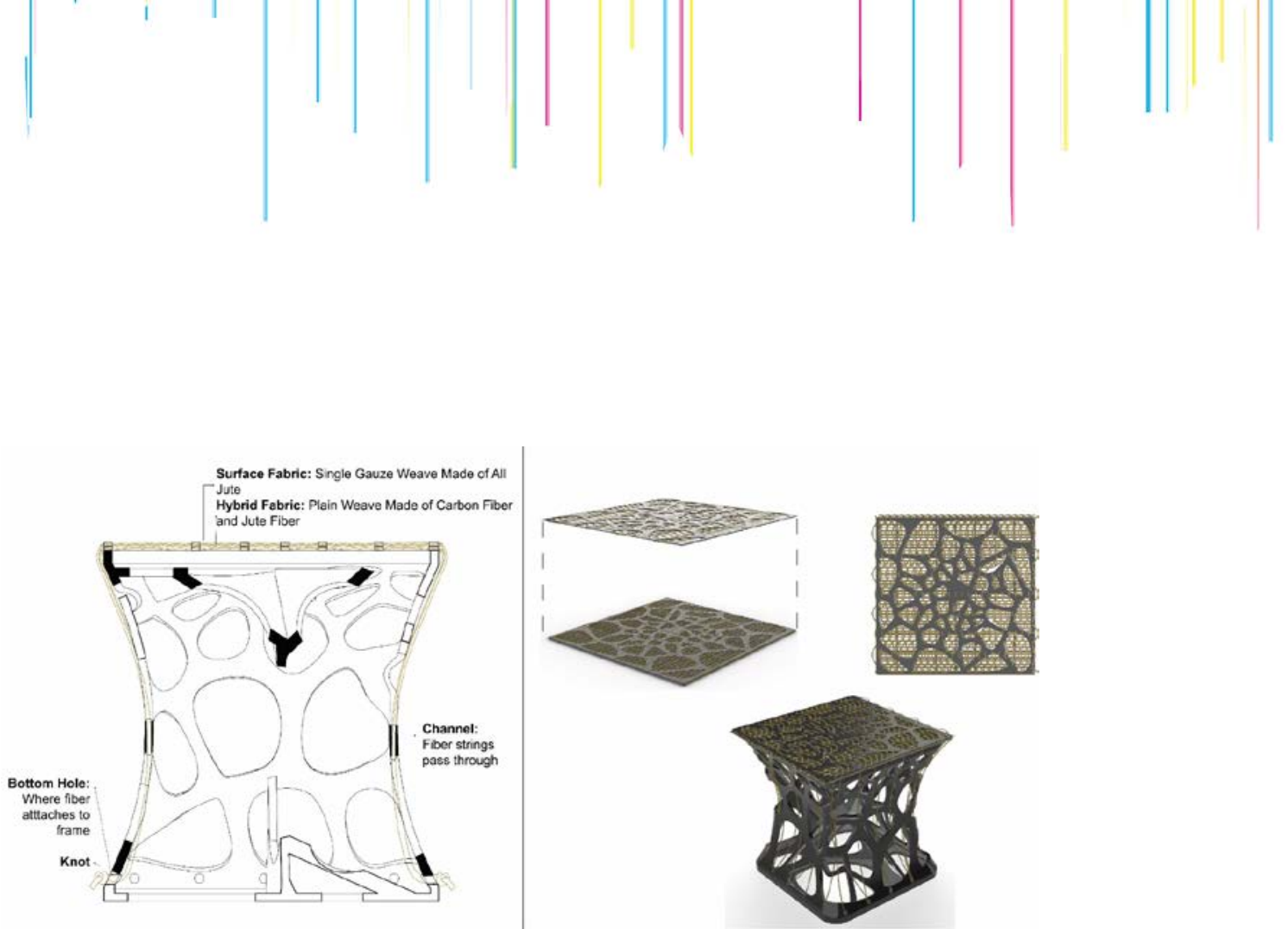

Figure 6. Fabrication Details- Textile Attachment to the Frame. Source: Berfin Evrim, 2021.

\section{Discussion}

In short, this research emphasizes problems about conventional fabrication techniques and limited digital platforms for textile design. For instance, manufacturing methods of fiber structures are mostly concentrated on using molding techniques, which require mold fabrication. This method causes a waste of material and time. Additionally, chemical resins are applied to stiffen fabric structures that have various negative environmental and health impacts. As a solution, this study presents a fabrication method that integrates traditional weaving and 3D printing techniques and a digital tool.

The other problem is a lack of digital platforms for designers to model their textile design and test it structurally. Therefore, a generative algorithm is built within Karamba 3D to provide a parametric platform to model the fabrics and $3 \mathrm{D}$ printed patterns to test the structural resistance of the 3D printed woven textiles. This digital tool allows designers to simulate 3D modeled textiles under an applied load. As a result of the developed digital tool, designers can reduce the amount of material used to construct architectural and structural elements such as barrier walls, columns, beams, structural ceiling, and, indeed, furniture construction which has woven hybrid fabric structure.

The limitation of this framework is that Karamba 3D has limited libraries for material selection. Hence, material properties are edited manually for more accurate results. The other issue with the tool is that it does not convert curves into polylines that become structural elements. Therefore, the warp and weft curves are transformed into polylines for finite element analysis.

The stool design reflects the complex relationship between horizontal and vertical elements. Bone structure is studied to convert the complexity into the continuity of these two elements by allowing the seating surface to stay in 
tension by going through a 3D printed vertical stool frame. Trabecular bone provides a formal shape and structural method into the stool design. The geometry of the 3D printing pattern is inspired by the microscopic Voronoi structure of trabecular bone. Even though the microscopic geometries are not translated into woven textile directly, the concept of using graded material is derived from bone structure to add more carbon fiber row when it is needed.

Overall, the integrative fabrication method of weaving and 3D printing improves the use of textiles in architecture. For instance, the 3D printed woven fabrics can be used as ceiling and floor where the walls are 3D printed of concrete or earth. The strings can go through in between walls and attach to the vertical elements. Furthermore, the 3D printed fabrics can be used as formwork for 3D printing walls, shells, and roofs.

Acknowledgements. The author would like to acknowledge the contributions of Felecia Davis, Shadi Nazarian, and Charles Bakis in this project. They provided critical guidance during the research project. The study is conducted at SCDC, Pennsylvania State University.

\section{References}

Aakash, A., Nasir, M. A., Khalid, M. Y., Nauman, S., Shaker K., Khushnood S., Altaf K., Zeeshan M., \& Hussain, A. (2019). Experimental and Numerical Characterization of Mechanical Properties of carbon/jute Fabric Reinforced Epoxy Hybrid Composites. Journal of Mechanical Science and Technology 33 (9): 4217-4226.

Clemens Preisinger. (2020). Karamba 3D (version 1.3.3) [Computer software]. https://www.karamba3d.com/

Estrada, R. D., Wyller, M, \& Dahy, H. (2019). Aerochair: Integrative Design Methodologies for Lightweight Carbon fiber Furniture Design. In J. S. Pedro, G. C. Henriques, \& J. P. Xavier (Eds.), Proceedings of 37 eCAADe and XXIII SIGraDi Joint Conference (pp. 691-702). São Paulo: Blucher. DOI 10.5151/proceedingsecaadesigradi2019_665

lida,H., Fujishima, T., Ohbuchi, Y.,\& Sakamoto, H. (2014). Application of New Design Method by High-Strength Composite Material. Applied Mechanics and Materials 607: 915-919.

Kycia, A., \& Guiducci, L. (2020). A material platform for digitally designed, materialinformed surface elements. In L. Werner,\& D. Koering,(Eds.), Proceedings of the 38th eCAADe Conference (pp. 21-30), TU Berlin, Berlin, Germany

Martínez, J., Song, H., Dumas J., \& Lefebvre, S. (2017). Orthotropic k -Nearest Foams for Additive Manufacturing. ACM Transactions on Graphics (TOG) 36 (4): 1-12.

Nan, I. C., Patterson, C., \& Pedreschi, R. (2019). Emerging Territories of Digital Material Practice. In RTD2017 Proceedings of the 3rd Biennial Research Through Design Conference RTD. https://doi.org/10.6084/m9.figshare.4747000.v1 
Oxman, N. (2010). Structuring Materiality: Design Fabrication of Heterogeneous Materials. Architectural Design 80 (4): 78-85.

Wang, X., Tam, K.-M. M., Beaudouin-Mackay, A., Hoyle, B., Mason, M., Guo, Z., Gao, W., Li, C., Zhu, W., Karsan, Z., Kao, G. T.-C., Zhang, L., Chai, H., Yuan, P. F., \& Block, P. (2020). 3d-Printed Bending-Active Formwork for Shell Structures. In P. F. Yuan, M. Xie, N. Leach, J. Yao, \& X. Wang (Eds.), Architectural Intelligence: Selected Papers from the 1st International Conference on Computational Design and Robotic Fabrication (CDRF 2019) (pp. 295-314). Springer Singapore. https://doi.org/10.1007/978-981-15-6568-7_18 